\title{
A Nonlinear Master Equation for a Degenerate Diffusion Model of Biofilm Growth ${ }^{\star}$
}

\author{
Hassan Khassehkhan ${ }^{1}$, Thomas Hillen ${ }^{2}$, and Hermann J Eberl ${ }^{1}$ \\ 1 Dept. of Mathematics and Statistics, \\ University of Guelph Guelph, ON, Canada, N1G 2W1 \\ $\{$ hkhasseh, heberl\} @uoguelph.ca \\ 2 Dept. of Mathematical and Statistical Sciences, Centre for Mathematical Biology \\ Univ. of Alberta, Edmonton, AB, Canada, T6G 2G1 \\ thillen@math.ualberta.ca
}

\begin{abstract}
We present a continuous time/discrete space model of biofilm growth, starting from the semi-discrete master equation. The probabilities of biomass movement into neighboring sites depend on the local biomass density and on the biomass density in the target site such that spatial movement only takes place if (i) locally not enough space is available to accommodate newly produced biomass and (ii) the target site has capacity to accommodate new biomass. This mimics the rules employed by Cellular Automata models of biofilms. Grid refinement leads formally to a degenerate parabolic equation. We show that a set of transition rules can be found such that a previously studied ad hoc density-dependent diffusion-reaction model of biofilm formation is approximated well.
\end{abstract}

\section{Introduction}

Most bacterial populations live as microbial depositions on immersed surfaces (called substratum in the biofilm context). These biofilm colonies are not a consequence of active or even deliberate aggregation of microorganisms but of immobilization and cell division. Once cells become sessile they start to produce an extracellular polymeric network in which they are themselves embedded (EPS). Thus, they are heavily restricted in their mobility. Living in biofilm colonies is quite different from living as a suspended population. For example, the colony offers protection against mechanical washout or antibiotic attacks.

As long as the conditions are locally favorable to sustain microbial growth, cells will increase in size and eventually divide. The local expansion of a biofilm is primarily driven by this growth process. As new cells require more space, the neighbors must make way. The bacteria closer to the food source grow faster than the bacteria further away, e.g. the ones close to the substratum. This can lead to the characteristic "biofilm mushroom structures" [10] which appear as if the biofilm is growing toward the region with higher food concentrations. In

\footnotetext{
* Supported by the Advanced Foods and Materials Network (AFMNET) [HJE] and the Natural Science and Engineering Research Council of Canada (NSERC) [TH].
} 

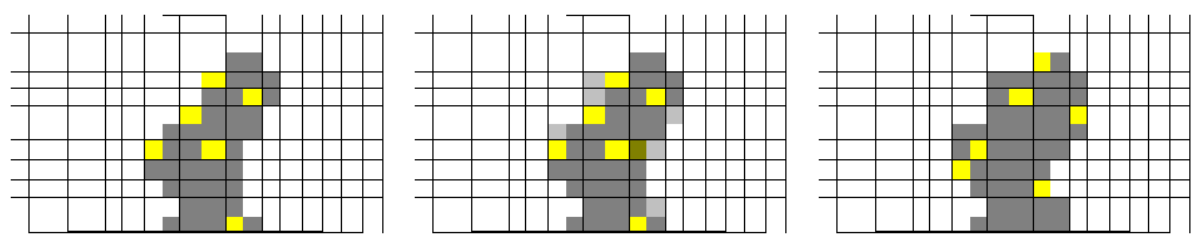

Fig. 1. Schematics of one time step of biomass spreading in cellular automata models, cf [12 13], (left to right): If the biomass in a grid cell approaches or exceeds maximum packing density (yellow grid cells), mass is moved into neighboring sites. If empty neighboring grid cell are found the excess biomass is placed there (grey), otherwise re-shuffling takes place (existing biomass is placed in a neighboring cell [grey-yellow], the excess biomass of which is placed in a suitable empty site).

contrast to chemotaxis, however, this is not an active movement up the nutrient gradient but a consequence of the fact that life under conditions of abundance is more conducive to population growth than under conditions of starvation.

The most widespread technique in modern biofilm modeling, and in fact the modeling concept that first was used to describe multi-dimensional biofilm formation are cellular automata, e.g. 27/2 1314, cf Fig. 1] Deterministic continuum models include the density-dependent diffusion model [3], which is formulated as a traditional spatially structured population and resource dynamics model. Despite the good phenomenological agreement between different model approaches [10 17] no attempts have been made so far to relate these seemingly so different models to each other. We formulate here a discrete space, continuous time model, starting from the master equation that describes the probability that bacteria move from one site on a regular lattice into a neighboring site and vice versa. This movement follows stochastic rules which qualitatively mimic those in 12 . The probability of biomass movement into neighboring sites has to account for two particular aspects: (i) It depends on the availability of space in that site (volume filling, cf [11]), and (ii) as long as there is capacity to accommodate new biomass locally the incentive to move into a neighboring site is small (quenching). Semi-discrete master equation models lend themselves in an often straightforward manner to deriving deterministic continuous models. Our goal is to derive from this spatially discrete description the phenomenological nonlinear diffusion model [3]. In fact, since this was originally introduced in an ad hoc fashion, the semi-discrete approach described here can be understood as an a posteriori derivation of this model.

Semi-discrete master equation models for spatially structured populations have been developed for many different types of migration in ecology and cell biology, such as aggregation and (chemo-)taxis, cf. [191116. The advantage of the approach is that phenomenological migration rules are easily formulated based on assumptions on the individual level, while the well-developed machinery of differential equations can be used to study the model on the population level. The biofilm model presented here has qualitative properties that are distinct from other master equation models, since it has to account for the properties (i) 
and (ii) above. Moreover, since the driving force behind spatial expansion of the biofilm is population growth, this needs to be included in every biofilm model.

\section{The Spatially Discrete Master Equation}

We consider an equidistant discretization of the real line and denote by $\mathcal{T}_{i}^{ \pm}$the probability that biomass moves from the $i$ th grid cell into neighbor cell $i \pm 1$, cf. Fig. 2, We follow common practice and equate the dependent variable $u_{i}$ with the population density in the $i$ th site on the lattice. Like most biofilm models, we subsume EPS and cells in this variable. Since the site's capacity to accommodate bacteria is limited, we normalize the density with respect to the maximum density, i.e. we interpret it as the volume fraction of site $i$ occupied by the population. Thus, $0 \leq u_{i} \leq 1$ is mandatory. The $\mathcal{T}_{i}^{ \pm}$can also be understood as mass transfer rates. The master equation reads

$$
\frac{d u_{i}}{d t}=\mathcal{T}_{i-1}^{+} u_{i-1}+\mathcal{T}_{i+1}^{-} u_{i+1}-\left(\mathcal{T}_{i}^{+}+\mathcal{T}_{i}^{-}\right) u_{i}+\mathcal{K}_{i} u_{i},
$$

where $\mathcal{T}_{i}^{ \pm}=\alpha q_{i}^{ \pm}$with a scaling factor $\alpha$ that depends on time-scale and length scale, i.e. distance between two sites $h$. More specifically, for diffusion problems $\alpha$ scales with $h^{2}$, such that $\lim _{h \rightarrow 0} \alpha h^{2}=\alpha_{0}>0$. The probability $q_{i}^{ \pm}$of a jump from location $i$ to location $i \pm 1$ depends on the densities in both sites, i.e. on $u_{i}$ and on $u_{i \pm 1}$. We make the general ansatz

$$
q_{i}^{ \pm}=q\left(u_{i}\right) p\left(u_{i \pm 1}\right)
$$

In cellular automaton biofilm models like [12], spatial spreading of biomass takes place if the local biomass density $u_{i}$ reaches or exceeds the maximum cell packing density, i.e. $u \approx 1$. Then a given or randomly chosen amount of the local biomass density is placed in a neighboring grid cell according to some stochastic local rule. In the continuum model [3], on the other hand, the spatial spreading is described deterministically: biomass is not moved into neighboring sites as long as newly produced biomass still easily fits into the local site. As $u_{i}$ increases and gets close to $1, \mathcal{T}_{i}^{ \pm}$increases. Moreover, the bigger the volume fraction in the target site, the smaller is $\mathcal{T}_{i}^{ \pm}$. Thus, we assume $q(u)$ to be a monotonously increasing function and $p(u)$ to be a monotonously decreasing function. Assuming sufficient smoothness, we have for $p, q$ the properties

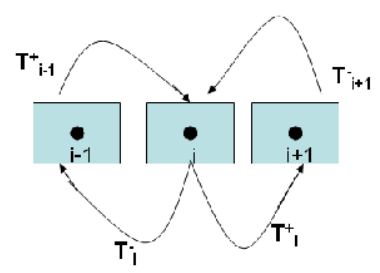

Fig. 2. Schematic of movement of biomass between neighboring lattice cells. The probability for biomass to move from cell $i$ into cell $i \pm 1$ is denoted by $\mathcal{T}_{i}^{ \pm}$etc. 


$$
\begin{aligned}
& q(0)=0, \quad q^{\prime}(u) \geq 0, \quad q(1)=1, \\
& p(0)=1, \quad p^{\prime}(u) \leq 0, \quad p(1)=0 \text {. }
\end{aligned}
$$

In (1), $\mathcal{K}_{i}$ is the net biomass production rate in grid cell $i$. This is the actual force driving the expansion of biofilm colonies. If $\mathcal{K}_{i}$ is a positive constant the model describes unrestricted exponential growth. In most biofilm systems, growth is eventually not everywhere unlimited. Limitations can be induced by local limitation of nutrients, iron, oxygen (aerobic case) etc. or by large amounts of growth inhibitors such as protonated lactic acids, proton ions [i.e. low $\mathrm{pH}$ ], or oxygen (anaerobic case). Modeling these effects is conceptually straightforward. Each growth limiting substrate is described by an additional transport-reaction equation, which is coupled with (1) in the reaction term $\mathcal{K}_{i}$. The growth limitation mechanisms listed above are due to an increase of biomass in the system. For example, the more bacteria there are in the system the more nutrients are depleted, etc. In many biofilm systems the net growth rate can become negative, e.g if nutrients are locally completely depleted and natural cell loss dominates. Under this light, assuming $\mathcal{K}_{i}=$ const is the most challenging case because biomass production is highest and, thus, biomass spreading most pronounced.

The relationship between the spatially discrete and a continuous description is established by passing the grid cell size $h$ in (1) to the continuous limit, $h \rightarrow 0$. Assuming enough smoothness we approximate $q\left(u_{i \pm 1}\right)$ and $p\left(u_{i \pm 1}\right)$ in the usual way by Taylor polynomials about $u_{i}$,

$$
\begin{aligned}
& q\left(u_{i \pm 1}\right)=q\left(u_{i}\right)+\left(u_{i \pm 1}-u_{i}\right) q^{\prime}\left(u_{i}\right)+\left(u_{i \pm 1}-u_{i}\right)^{2} q^{\prime \prime}\left(u_{i}\right) / 2+\mathcal{O}\left(\left(u_{i \pm 1}-u_{i}\right)^{3}\right) \\
& p\left(u_{i \pm 1}\right)=p\left(u_{i}\right)+\left(u_{i \pm 1}-u_{i}\right) p^{\prime}\left(u_{i}\right)+\left(u_{i \pm 1}-u_{i}\right)^{2} p^{\prime \prime}\left(u_{i}\right) / 2+\mathcal{O}\left(\left(u_{i \pm 1}-u_{i}\right)^{3}\right) .
\end{aligned}
$$

Interpreting $u_{i}$ as a quantity in the cell center $x_{i}$, we can interpolate the grid function $u_{i}$ by a continuous function $u$ with $u\left(t, x_{i}\right)=u_{i}(t)$. For given $t$ we approximate $u\left(t, x_{i \pm 1}\right)$ by Taylor polynomials

$$
u\left(t, x_{i \pm 1}\right)=u_{i}(t) \pm h \frac{\partial u_{i}(t)}{\partial x}+\frac{h^{2}}{2} \frac{\partial^{2} u_{i}(t)}{\partial x^{2}}+\mathcal{O}\left(h^{3}\right)
$$

where $\frac{\partial u_{i}}{\partial x}$ is short-hand for $\frac{\partial u}{\partial x}\left(\cdot, x_{i}\right)$. Substituting these expressions into (1) we obtain after dropping $\mathcal{O}\left(h^{3}\right)$ terms and sorting by powers of $\partial u_{i} / \partial x$

$$
\begin{aligned}
\frac{\partial u_{i}}{\partial t}=\mathcal{K} u_{i} & +\alpha h^{2}\left[p\left(u_{i}\right) q\left(u_{i}\right)+u\left(p\left(u_{i}\right) q^{\prime}\left(u_{i}\right)-q\left(u_{i}\right) p^{\prime}\left(u_{i}\right)\right)\right] \frac{\partial^{2} u_{i}}{\partial x^{2}}+ \\
& +\alpha h^{2}\left[p\left(u_{i}\right) q^{\prime \prime}\left(u_{i}\right) u_{i}+2 q^{\prime}\left(u_{i}\right) p\left(u_{i}\right)-u_{i} q\left(u_{i}\right) p^{\prime \prime}\left(u_{i}\right)\right]\left(\frac{\partial u_{i}}{\partial x}\right)^{2} .
\end{aligned}
$$

Defining now the density dependent diffusion coefficient

$$
D(u):=p(u) q(u)+u\left(p(u) q^{\prime}(u)-q(u) p^{\prime}(u)\right)
$$

equation (4) can be written as a diffusion-reaction equation in divergence form

$$
\frac{d u}{d t}=\frac{\partial}{\partial x}\left(D(u) \frac{\partial u}{\partial x}\right)+\mathcal{K} u .
$$




\section{An Inverse Problem}

A density-dependent diffusion-reaction model for biofilm formation was introduced in 3 and has been studied since numerically and analytically in its original and extended versions, e.g. in 4568. The pecularity of that model lies in the form of the nonlinear diffusion coefficient $D(u)$, namely

$$
D(u)=\delta u^{a}(1-u)^{-b}, \quad a, b \geq 1 .
$$

For small $u \ll 1$ this type of nonlinear diffusion acts like the porous medium equation, while for large $u \approx 1$ it shows fast diffusion effects. The interplay of both effects is required to describe a growing biofilm. It was shown in 6 , that solutions of this model remain bounded by and separated from unity, $u<1-\eta$, for a $\eta>0$, as long as the biofilm/water interface stays away from the boundary of the domain somewhere. Thus, the singularity is never reached. It follows then with standard arguments about degenerate diffusion-reaction equations, cf [15], that initial data with compact support imply solutions with compact support. In our application this means that the interface between biofilm and surrounding liquid is sharp and propagates at finite speed.

We try to find jump probabilities $q(u)$ and $p(u)$ with (3) such that the diffusion-reaction model (6) with (7) is recovered. This is a constrained scalar boundary value problem for two unknown functions. It is transformed into an ordinary boundary value problem by introducing one more assumption on $q$ and/or $p$. We propose

$$
p(u):=1-u q(u)
$$

It is easily verified that $p$ satisfies (3) if $q$ does and vice versa. With (8) equation (5) becomes the linear ordinary differential equation

$$
u q^{\prime}(u)+q(u)=D(u),
$$

which degenerates for $u=0$. Moreover, the right hand side of (9) blows up for $u=1$. We introduce the following regularization with small parameter $\epsilon>0$, which was already used in the analysis of the biofilm model (6), (7) in 688.

$$
D_{\epsilon}(u)=\left\{\begin{array}{lll}
\delta \frac{(u+\epsilon)^{a}}{(1-u)^{b}}, & \text { for } & u<1-\epsilon, \\
\delta \epsilon^{-b}, & \text { for } & u \geq 1-\epsilon .
\end{array}\right.
$$

The unique solution $q_{\epsilon}$ of the regularized initial value problem

$$
(u+\epsilon) q^{\prime}(u)+q(u)=D_{\epsilon}(u), \quad q(0)=0
$$

is obtained as the strictly increasing function

$$
q_{\epsilon}(u)=\frac{1}{u+\epsilon} \int_{0}^{u} D_{\epsilon}(s) d s,
$$

which is bounded for every $\epsilon>0$ and $0 \leq u \leq 1$. Moreover, $1<q_{\epsilon}(1)<\infty$ for every small enough $\epsilon$. Thus, continuity and monotonicity imply that there exists 
a $\tilde{u}_{\epsilon}<1$ such that $q_{\epsilon}\left(\tilde{u}_{\epsilon}\right)=1$, while $q_{\epsilon}(u)<1$ for $u<\tilde{u}_{\epsilon}$ and $q_{\epsilon}(u)>1$ for $u>\tilde{u}_{\epsilon}$. Hence, $q_{\epsilon}(u)$ does not belong to the desired class of functions

$$
\mathcal{G}:=\left\{g \in \mathcal{C}([0,1]): g(0)=0, \quad g(1)=1, \quad g\left(u_{1}\right) \leq g\left(u_{2}\right) \quad \forall u_{1}<u_{2}\right\} .
$$

Thus the problem of finding an exact solution of (11) in $\mathcal{G}$ is ill-posed and the best we can hope for is to find a function $r_{\epsilon}(u) \in \mathcal{G}$ that solves (11) approximately. Thus, we are looking for the function in $\mathcal{G}$ with the smallest distance from $q_{\epsilon}$,

$$
\min _{r \in \mathcal{G}}\left\|q_{\epsilon}-r\right\|_{2}
$$

This minimization problem is solved by the function

$$
r_{\epsilon}(u)= \begin{cases}q_{\epsilon}(u), & \text { for } \quad u \leq \tilde{u}_{\epsilon} \\ 1, & \text { for } u>\tilde{u}_{\epsilon}\end{cases}
$$

because $r_{\epsilon} \in \mathcal{G}$ and

$$
\left\|q_{\epsilon}-r_{\epsilon}\right\|_{2}^{2}=\int_{0}^{\tilde{u}_{\epsilon}}\left(q_{\epsilon}(s)-r_{\epsilon}(s)\right)^{2} d s+\int_{\tilde{u}_{\epsilon}}^{1}\left(q_{\epsilon}(s)-r_{\epsilon}(s)\right)^{2} d s .
$$

The first integral vanishes for $r(u)=r_{\epsilon}(u)$ as defined above, and, since $q_{\epsilon}(u)>1$ and $r(u) \leq 1$ for $u>\tilde{u}_{\epsilon}$, the second integral becomes minimal if $r(u)=1$ for $u>\tilde{u}_{\epsilon}$. Passing the regularization parameter to the limit, $\epsilon \rightarrow 0$, then $r_{\epsilon}(u) \rightarrow r(u)$ pointwise, where

$$
r(u)=\left\{\begin{array}{ll}
\phi(u), & \text { for } \quad u \leq \tilde{u}, \\
1, & \text { for } \quad u>\tilde{u},
\end{array} \quad \text { with } \quad \phi(u)=\frac{1}{u} \int_{0}^{u} D(s) d s .\right.
$$

Function $\phi(u)$ is the strictly increasing solution of the initial value problem (9) with $q(0)=0$ and $\tilde{u}$ is the unique value such that $\phi(\tilde{u})=1$. Note that $\lim _{u \rightarrow 1} \phi(u)=\infty$. Obviously $r \in \mathcal{G}$. We choose $q(u):=r(u)$. It remains to validate how good this approximation is for practical purposes. We recall from [6] that the solution of (26) with (7) satisfies $u<1-\eta$ for a $\eta>0$, which depends on the parameters and the initial data. Thus, if $\tilde{u}>1-\eta$ then (6) with (7) is equivalent with the PDE model that one obtains from the lattice model with jump probability $q(u)=r(u)$ from (14). Otherwise they are equivalent as long as the solutions remain bounded by $\tilde{u}$, i.e. for a finite time interval. Unfortunately, the proof in [6] does not allow to compute quantitative estimates for $\eta$, so that we have to fall back on comparisons with computer simulations. For example, in the $2 \mathrm{D}$ and $3 \mathrm{D}$ simulations of the single-species growth model in [456], the solution reached values above $u \approx 0.99$ but remained below $u \approx 0.9999$, thus, we have the numerical estimates $\hat{\eta}>10^{-4}$. In all three cases the exponents in the biomass diffusion coefficient were $a=b=4$ and the biofilm motility coefficient $\delta<10^{-12}$. Then

$$
\int_{0}^{u} D(s) d s=u \phi(u)=\delta\left(u+4 \ln (1-u)-\frac{-18 u^{2}+30 u-13}{3(1-u)^{3}}-\frac{13}{3}\right) .
$$




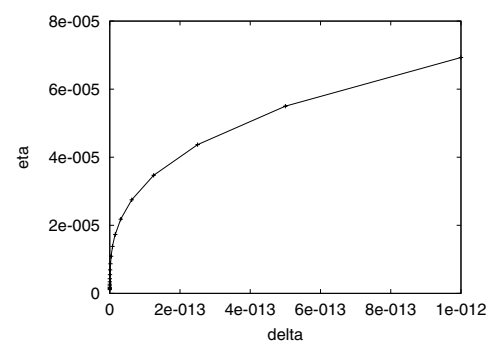

Fig. 3. $\tilde{\eta}=1-\tilde{u}$ as a function of the biomass mobility parameter $\delta$

On the other hand the value $\tilde{\eta}:=1-\tilde{u}$ can be computed from (15). It is plotted in Fig. 3 for $\delta$ covering several orders of magnitude. $\tilde{\eta}$ decreases as $\delta$ decreases. In all cases, $\tilde{u}$ remained above the maximum values of $u$ in the numerical simulations reported above, i.e $\hat{\eta}>1-\tilde{u}=: \tilde{\eta}$ by at least one order of magnitude. Thus the nonlinear diffusion-reaction model that is derived from the semi-discrete master equation (1) with the jump probabilities $T_{i}^{ \pm}(u)=\alpha q\left(u_{i}\right) p\left(u_{i \pm 1}\right), q(u):=$ $r(u), p(u):=1-u q(u)$ is in these simulations indeed equivalent to the doubledegenerate density-dependent diffusion-reaction model.

We remark that (6) can also be written in the Laplacian form

$$
u_{t}=\frac{\partial^{2}}{\partial x^{2}}\left(\int_{0}^{u} D(s) d s\right)+\mathcal{K} u .
$$

Hence,(1) is equivalent with the finite difference discretisation of (16)

$$
\frac{d u_{i}}{d t}=\alpha\left(u_{i-1} q\left(u_{i-1}\right)-2 u_{i} q\left(u_{i}\right)+u_{i+1} q\left(u_{i+1}\right)\right)+\mathcal{K} u_{i} .
$$

Note that assuming $p(u) \equiv 1$ instead of (8), i.e. transition probability does not depend on density in target site, also leads to (9) and thus to (17).

\section{Numerical Results}

We conduct computer simulations of the semi-discrete model in two space dimensions on a $n \times m$ grid. Nutrients are assumed to be nowhere limited, $\mathcal{K}=$ const. In interior grid points $1<i<n, 1<j<m$ the $2 \mathrm{D}$ variant of (1) reads

$$
\begin{aligned}
\frac{d}{d t} u_{i, j}=\mathcal{K} u_{i, j} & +\mathcal{T}_{i-1, j}^{+, 0} u_{i-1, j}+\mathcal{T}_{i+1, j}^{-, 0} u_{i+1, j} \mathcal{T}_{i, j-1}^{0,+} u_{i, j-1}+\mathcal{T}_{i, j+1}^{0,-} u_{i, j+1} \\
& -\left(\mathcal{T}_{i, j}^{+, 0}+\mathcal{T}_{i, j}^{-, 0}+\mathcal{T}_{i, j}^{0,+}+\mathcal{T}_{i, j}^{0,-}\right) u_{i, j},
\end{aligned}
$$

where $\mathcal{T}_{i, j}^{ \pm, 0}=\alpha q\left(u_{i, j}\right) p\left(u_{i \pm 1, j}\right)$ and $\mathcal{T}_{i, j}^{0, \pm}=\alpha q\left(u_{i, j}\right) p\left(u_{i, j \pm 1}\right)$. The derivation of the $2 \mathrm{D}$ equivalents of (41) and (6) follows the same procedure as in 1D, albeit notation and calculations are more cumbersome. The inverse problem in Section 3 is independent of the problem dimension. 

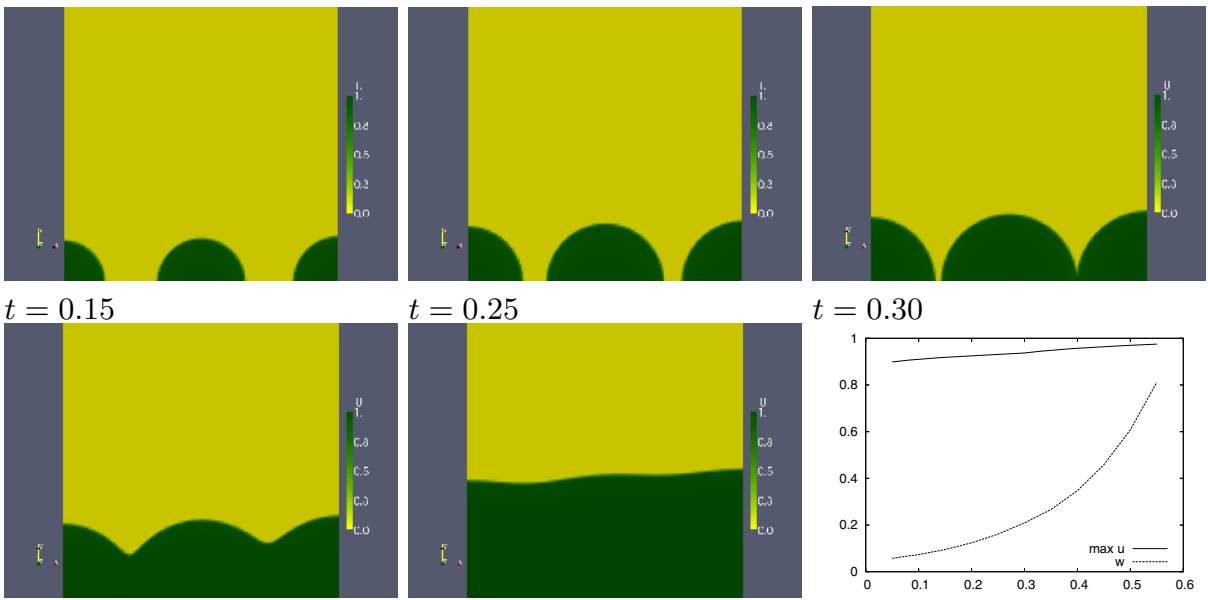

$t=0.30$

$t=0.35$

$t=0.45$

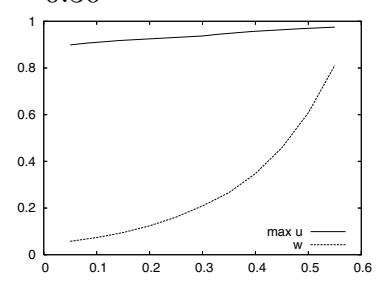

Fig. 4. 2D Simulation of biofilm growth on a $128 \times 128$ grid. Shown are for selected time instances $t$ the biomass densities $u_{i, j}$. The bottom right insert shows $\max _{i j} u_{i j}(t)$ and the occupancy function $\omega(t)$, i.e. the fraction of grid cells with $u>10^{-6}$.

In $i=1, i=n, j=1, j=m$, (18) is corrected such that no exchange of biomass across the lattice boundaries takes place, mimicking homogeneous Neumann conditions. We set in the "virtual grid points" $u_{0, j}:=u_{1, j}, u_{n+1, j}:=$ $u_{n, j}, u_{i, 0}:=u_{i, 1}, u_{i, m+1}:=u_{i, m}$ and eliminate them from (18). The system of $\mathrm{nm}$ ordinary differential equations is integrated by the Runge-Kutta-Fehlberg method RKF4(5).

We use a $128 \times 128$ grid. Cells are inoculated in three spherical pockets around the grid cells $(i, j)=(1,1)$ [radius $\rho=0.1 n$, initial biomass density $u_{0}=0.8$ ], $(i, j)=(n / 2,1)\left[\rho=0.11 n, u_{0}=0.75\right]$, and $(i, j)=(n, 1)\left[\rho=0.12 n, u_{0}=0.7\right]$. We take $q(u)$ from (14) and $p(u)=1-u q(u)$. For the coefficients of $D(u)$ we pick $a=b=4$ and $\delta=10^{-12} \mathrm{~m}^{2} / d$, for the biomass production rate $\mathcal{K}=6 / d$. With a simulation domain of $100 \times 100 \mu \mathrm{m}^{2}, h$ corresponds to $0.78125 \mu \mathrm{m}$.

In Fig. 4 we show for selected $t$ the biomass density $u_{i, j}(t)$, interpolated between grid cells by the visualization software. Initially the three colonies

Table 1. Results of a grid refinement study. Reported are the values $N_{1}:=$ $\frac{1}{n m} \sum_{i, j}\left|u_{i j}\left(t_{p}\right)-\tilde{u}_{i j}\left(t_{p}\right)\right|$ for three selected time steps, where $\tilde{u}$ is the projection of the coarser grid onto the finer grid.

\begin{tabular}{l|ccc} 
grid refinement & $t=0.15$ & $t=0.25$ & $t=0.30$ \\
\hline$h=1 / 16 \rightarrow 1 / 32$ & 0.025910 & 0.046667 & 0.056930 \\
$h=1 / 32 \rightarrow 1 / 64$ & 0.010767 & 0.017459 & 0.021014 \\
$h=1 / 64 \rightarrow 1 / 128$ & 0.003537 & 0.005473 & 0.006467 \\
$h=1 / 128 \rightarrow 1 / 256$ & 0.001828 & 0.002741 & 0.003442 \\
$h=1 / 256 \rightarrow 1 / 512$ & 0.000873 & 0.001184 & 0.001365
\end{tabular}




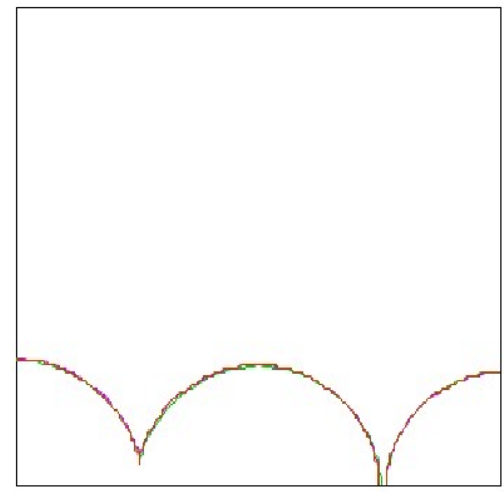

Fig. 5. Biofilm/water interface at $t=30$ for grid sizes $h=1 / 128,1 / 256,1 / 512$ [green, magenta, brown]

solidify, i.e. $u$ increases, first without notable expansion. Later, the colonies expand spherically. Eventually, neighboring colonies merge and a homogeneous thick layer of biomass develops that expands exponentially. This is a consequence of the abundance of food and agrees with all other biofilm models. The simulation is stopped before the entire lattice is filled with biomass. The biomass density remains clearly below the cut-off value $\tilde{u}$ that was computed above.

In order to investigate how the solution of the spatially discrete model changes with grid refinement, we repeat and compare the simulations on grids of size $2^{k} \times 2^{k}$, where $k=4, \ldots, 9$. Table 1 shows the convergence of the grid refinement study. This is visually supported by Fig. 5 where the biofilm/water interface is plotted for subsequent grids. These agree well within plotting accuracy.

\section{Conclusion, Discussion, and Future Work}

Spatially discrete master equations are routinely used to derive population models that can be studied with the well developed machinery of partial differential equations. We use this approach to derive a highly nonlinear diffusion-reaction model of biofilm expansion. The underlying mechanisms are quite different than for other biological systems. Most notably, spatial spreading of biomass is driven by production of new biomass and takes place only if locally no space is available to accommodate newly produced cells. In the continuous limit this is rendered by a combination of degenerate and fast diffusion. In numerical simulations we could show that the spatially discrete master equation and the continuous model agree well. The assumptions that we made to define the probabilities for biomass movement mimic the stochastic rules that are used in cellular automata biofilm models. Therefore, we understand the master equation model as a link between discrete stochastic and continuous deterministic biofilm models.

In our current study the equivalence of spatially discrete and continuous model rests on empirical computer simulations. While it follows from continuity that both models agree exactly for some time, the question how long this equivalence 
is valid certainly warrants more rigorous mathematical analysis. On the modeling side, future research will involve the extension of the approach to mixed-culture biofilm systems and to biofilm systems with preferred spreading directions, e.g. biofilms that predominantly creep over the substratum rather than forming patchy biofilm colonies. Moreover, for realistic applications the biofilm population models need to be coupled with models of resource dynamics, as already indicated above.

\section{References}

1. Anguige, K., Schmeiser, C.: A one-dimensional model of cell diffusion and aggregation, incorporating volume filling and cell-to-cell adhesion. J. Math. Biol. (in press)

2. Chambless, J.D., Hunt, S.M., Stewart, P.S.: A three-dimensional computer model of four hypothetical mechanisms protecting biofilms from antimicrobials. Appl. Env. Microbiol. 72(3), 2005-2013 (2006)

3. Eberl, H.J., Parker, D.F., van Loosdrecht, M.C.M.: A new deterministic spatiotemporal continuum model for biofilm development. J. Theor. Medicine 3(3), 161175 (2001)

4. Eberl, H.J., Demaret, L.: A finite difference scheme for a doubly degenerate diffusion-reaction equation arising in microbial ecology. El. J. Diff. Equ. CS 15, 77-95 (2007)

5. Eberl, H.J., Sudarsan, R.: Exposure of biofilms to slow flow fields: the convective contribution to growth and disinfections. J. Theor. Biol. 253(4), 788-807 (2008)

6. Efendiev, M.A., Zelik, S.V., Eberl, H.: Existence and longtime behavior of a biofilm model. Comm. Pure Appl. Analysis 8(2), 509-531 (2009)

7. Hermanowicz, S.W.: A simple $2 \mathrm{D}$ biofilm model yields a variety of morphological features. Math. Biosc. 169(1), 1-14 (2001)

8. Khassehkhan, H., Efendiev, M.A., Eberl, H.J.: A degenerate diffusion-reaction model of an amensalistic probiotic biofilm control system: Existence and simulation of solutions. Discr. Cont. Dyn. Sys. B (in press)

9. Lizana, M., Padorn, V.: A spatially discrete model of aggregating populations. J. Math. Biol. 38, 79-102 (1999)

10. Van Loosdrecht, M.C.M., Heijnen, J.J., Eberl, H.J., Kreft, J.U., Picioreanu, C.: Mathematical modelling of biofilm structures. Ant. v. Leeuwenhoek 81, 245-256 (2002)

11. Painter, K., Hillen, T.: Volume-Filling and Quorum Sensing in Models for Chemosensitive Movement. Can. Appl. Math. Quart. 10(4), 501-543 (2002)

12. Picioreanu, C., van Loosdrecht, M.C.M., Heijnen, J.J.: A new combined differentialdiscrete cellular automaton approach for biofilm modeling: Application for growth in gel beads. Biotech. Bioeng. 57(6), 718-731 (1998)

13. Pizarro, G., Griffeath, D., Noguera, D.: Quantitative cellular automaton model for biofilms. J. Env. Eng. 127(9), 782-789 (2001)

14. Pizaro, G.E., Texiera, J., Sepulveda, M., Noguera, D.: Bitwise implementation of a 2D cellular automata biofilm model. J. Comp. Civ. Eng. 19(3), 258-268 (2005)

15. Samarskii, A.A., Galaktionov, V.A., Kurdyumov, S.P., Mikhailov, A.P.: Blow-up in quasilienar parabolic equations. de Gruyter, Berlin (1995)

16. Visser, A.W.: Lagrangian modelling of plankton motion: From deceptively simple random walks to Fokker-Planck and back again. J. Mar. Sys. 70(3-4), 287-299 (2008)

17. Wanner, O., Eberl, H., Morgenorth, O., Noguera, D., Picioreanu, D., Rittmann, B., van Loosdrecht, M.: Mathematical Modelling of Biofilms. IWAPublishing, London (2006) 